\title{
Local chromatic number of quadrangulations of surfaces
}

\author{
Bojan Mohar*† \\ Department of Mathematics \\ Simon Fraser University \\ Burnaby, B.C. \\ mohar@sfu.ca
}

\author{
Gábor Simonyi ${ }^{\ddagger}$ \\ Alfréd Rényi Institute of Mathematics \\ Hungarian Academy of Sciences \\ Budapest, Hungary \\ simonyi@renyi.hu
}

\author{
Gábor Tardos ${ }^{\S}$ \\ Alfréd Rényi Institute of Mathematics \\ Hungarian Academy of Sciences, Budapest, Hungary \\ and \\ Department of Computer Science \\ Simon Fraser University, Burnaby, B.C. \\ tardos@renyi.hu
}

\begin{abstract}
The local chromatic number of a graph $G$, as introduced in [4], is the minimum integer $k$ such that $G$ admits a proper coloring (with an arbitrary number of colors) in which the neighborhood of each vertex uses less than $k$ colors. In [17] a connection of the local chromatic number to topological properties of (a box complex of) the graph was established and in [18] it was shown that a topological condition implying the usual chromatic number being at least four has a stronger consequence for the local chromatic number being at least four. As a consequence one obtains a generalization of the following theorem of Youngs [19]: If a quadrangulation of the projective plane is not bipartite it has chromatic number four. The generalization states that in this case the local chromatic number is also four.

Both papers [1] and [13] generalize Youngs' result to arbitrary non-orientable surfaces replacing the condition of the graph being not bipartite by a more technical condition of an odd quadrangulation. This paper investigates when these general results are true for the local chromatic number instead of the chromatic number. Surprisingly, we find out that (unlike in the case of the chromatic number) this depends on the genus of the surface. For the non-orientable surfaces of genus at most four, the local chromatic number of any odd quadrangulation is at least four, but this is not true for non-orientable surfaces of genus 5 or higher.
\end{abstract}

\footnotetext{
*Supported in part by an NSERC Discovery Grant, by the Canada Research Chair program, and by Research Grant P1-0297 of ARRS, Slovenia.

${ }^{\dagger}$ On leave from: IMFM \& FMF, Department of Mathematics, University of Ljubljana, Ljubljana, Slovenia.

${ }^{\ddagger}$ Research partially supported by the Hungarian Foundation for Scientific Research Grant (OTKA) Nos. K76088 and NK78439.

${ }^{\S}$ Supported in part by an NSERC Discovery Grant, by the Canada Research Chair program, and by Hungarian Foundation for Scientific Research Grant (OTKA) no. 78439.
} 
We also prove that face subdivisions of odd quadrangulations and Fisk triangulations of arbitrary surfaces exhibit the same behavior for the local chromatic number as they do for the usual chromatic number.

\section{Introduction}

We start by defining the terms in the title. By a surface we mean a compact connected 2manifold without boundary. A quadrangulation of a surface is a loopless graph on a surface with all faces being quadrilaterals. We allow parallel edges and quadrilateral faces with some of its vertices or even edges coinciding. Given an arbitrary orientation of all faces we can count the edges that break consistency of the orientation of the surface. (We say that consistency is not broken at some edge if, considering the orientation of a face as a closed walk along its boundary, we traverse the edge in opposite directions when considering the faces at its two sides.) Note that reversing the orientation of a face changes the status of its four edges (except for the edges appearing twice on the boundary) and thus the parity of the number of edges breaking consistency does not change. We call a quadrangulation even or odd depending on this parity. By the previous remark, the parity of the quadrangulation is determined by the quadrangulation itself and is independent of the actual orientation of the faces.

Note that every quadrangulation of an orientable surface is even as all faces can be oriented consistently.

We denote by $\chi(G)$ the chromatic number of the graph $G$. The following result was proved independently by Archdeacon et al. [1] and Mohar and Seymour [13].

Theorem $1.1([\mathbf{1}, \mathbf{1 3}])$ For an odd quadrangulation $G$ of a surface we have $\chi(G) \geq 4$.

The theorem only applies to non-orientable surfaces as orientable ones have no odd quadrangulations. Youngs [19] has established this earlier for the case of the projective plane. This result is simpler to state, since being even and being bipartite is equivalent for quadrangulations of the projective plane and since every quadrangulation of the projective plane is 4-colorable.

Theorem 1.2 ([19]) The chromatic number of a quadrangulation of the projective plane is either two or four.

In this paper we generalize the above results for the local chromatic number. The local chromatic number of a graph is defined in [4] as the minimum number of colors that must appear within distance 1 of a vertex. For the formal definition, let $N(v)=N_{G}(v)$ denote the neighborhood of a vertex $v$ in a graph $G$, that is, $N(v)$ is the set of vertices adjacent to $v$. For an integer $k \geq 1$, we call a proper vertex-coloring $c$ of a graph $G$ a local $k$-coloring if $|c(N(v))| \leq k-1$ for every vertex $v$ of $G$. The local chromatic number $\psi(G)$ of $G$ is the smallest $k$ such that $G$ has a local $k$-coloring.

The -1 term comes traditionally from considering "closed neighborhoods" $N(v) \cup\{v\}$ and results in a simpler form of the relations with other coloring parameters, like the trivial bound $\psi(G) \leq \chi(G)$.

More on the local chromatic number, including examples of graphs with arbitrarily high chromatic number and local chromatic number 3 , can be found in [4], see also [17, 18]. 
First we state the generalization of Youngs' theorem. This result was hinted in Remark 3 of [18], here we give a full proof for the sake of completeness in the next section.

Theorem 1.3 The local chromatic number of a quadrangulation of the projective plane is two or four.

Note that the non-orientable surfaces are determined by a positive invariant, their genus. The projective plane has genus one. Next we generalize Theorem 1.3 for higher genus surfaces. This is the main result of the present paper. Surprisingly, and unlike in the case of the chromatic number, the situation depends on the genus of the surface.

Theorem 1.4 (i) If $G$ is an odd quadrangulation of a (non-orientable) surface of genus at most four, then $\psi(G) \geq 4$.

(ii) Every non-orientable surface of genus at least five admits an odd quadrangulation that has a local 3-coloring using six colors.

We remark that at least five colors are needed to locally 3-color odd quadrangulations or in fact any graph with chromatic number higher than 3 . This is a consequence of the fact that any local 3-coloring with four colors can easily be transformed into a 3-coloring by changing the color of each vertex colored 4 to the color 1,2 , or 3 , which is not used in its neighborhood.

If we insist on five colors, the threshold lies higher:

Theorem 1.5 (i) If $G$ is an odd quadrangulation of a (non-orientable) surface of genus at most six, then $G$ has no local 3-coloring with at most five colors.

(ii) Every non-orientable surface of genus at least seven admits an odd quadrangulation that has a local 3-coloring using five colors.

For (some special cases of) Theorem 1.4(i) we give several proofs because they use very different approaches. In Section 2 we present the argument hinted in [18] for Theorem 1.3, i.e., for the projective plane. It is based on the concept of the hom space of a graph, which is just a slightly different version of the graph complexes known as box complexes, see [10], or those more generally called hom complexes, see (the cited second edition of) [9]. In Section 3 we give an algebraic proof for the non-existence of odd locally 3-chromatic quadrangulations that works for both the projective plane and the Klein bottle. In Section 4 we give a combinatorial argument that works for all surfaces of genus at most four, and henceforth proves Theorem 1.4(i). The results provided in Sections 2 and 3 follow also from the result of Section 4. But both of the former sections indicate connections between different concepts that are not shown by the combinatorial argument in Section 4. In Section 2 we use a general connection between any surface and the hom space of its quadrangulation. In Section 3 we show how an algebraic question about so-called semi-free groups is related to our graph theoretic problems. As a consequence of this connection and the existence of the quadrangulations claimed in Theorem 1.4 we can partially answer the algebraic question.

The locally 3-chromatic quadrangulations claimed in Theorems 1.4(ii) and 1.5(ii) are constructed in Section 5. These constructions are motivated by the argument in the preceding section. In Section 6 we prove Theorem 1.5(i). 
By inserting a vertex in each face (and joining it to the vertices on the boundary of that face) of an odd quadrangulation we obtain a triangulation that exhibits similar behavior for the chromatic number: any subgraph contained in a contractible part of the surface is 3-colorable, but the chromatic number of the whole graph is at least five. It is shown in Section 7 that the same holds for the local chromatic number. When coloring graphs on surfaces, another family of triangulations exhibits unusual behavior [14]; these are triangulations in which all vertices except two have even degree, and the two vertices of odd degree are adjacent. Fisk [5] proved that such triangulations cannot be 4-colored, and we show that the same holds for the local chromatic number. Finally, Section 8 contains related remarks and observations.

\section{Hom spaces}

In this section we prove Theorem 1.3 for which only a very rough argument was presented in [18]. We shall briefly introduce the necessary notation for hom spaces, but the reader unfamiliar with these concepts may consult [17] to find precise definitions and to see hom spaces in a broader context. We start with the definition of medial graphs that will be used in the sequel.

Let $G$ be a graph on a surface $S$. We define the medial graph $M(G)$, also embedded in $S$, as follows. The vertices of $M(G)$ correspond to edges of $G$. For an edge $e$ of $G$ we choose an interior point $v_{e}$ of $e$ as the corresponding vertex of $M(G)$. For each vertex $x$ of $G$ we consider the edges of $G$ incident to $x$ and place a cycle on the corresponding vertices of $M(G)$ as follows. Let $e_{1}, \ldots, e_{d}$ be the edges incident to $x$ listed in the cyclic order these edges leave $x$. For $1 \leq i<d$ we connect $v_{e_{i}}$ and $v_{e_{i+1}}$ with an edge of $M(G)$ drawn inside the face of $G$ bounded by $e_{i}$ and $e_{i+1}$. We connect $v_{e_{d}}$ and $v_{e_{1}}$ the same way.

Notice that $M(G)$ is a 4-regular graph embedded in $S$ that has two types of faces. The star faces are the ones containing a vertex $x$ of $G$ and bounded by the cycle we introduced on the vertices of $M(G)$ corresponding to the edges of $G$ incident to $x$ (the star of $x$ ). The cycle faces are the remaining faces of $M(G)$ : each is contained in a single face of $G$ and its vertices correspond to the edges on the corresponding facial walk.

The proof of Theorem 1.3 is based on the notion of the hom space $H(G)$ defined for any finite graph $G$. For the precise definition of this and related concepts see, e.g., [17]. Here we note that $H(G)$ is a cell complex, whose cells correspond to complete bipartite subgraphs of $G$. More precisely, the cells are of the form $A \uplus B$, where $A$ and $B$ are non-empty, disjoint sets of vertices, such that every vertex of $A$ is connected in $G$ to every vertex of $B .{ }^{1}$ The vertices of $H(G)$ are of the form $\{x\} \uplus\{y\}$, where $x y$ is an edge of $G$. In particular, every edge $x y$ gives rise to two vertices, $\{x\} \uplus\{y\}$ and $\{y\} \uplus\{x\}$, in $H(G)$. The map $\sigma$ that switches $A \uplus B$ with $B \uplus A$ is a continuous involution that makes $H(G)$ into a $\mathbb{Z}_{2}$-space. (For the notion and basic properties of $\mathbb{Z}_{2}$-spaces we also refer to [9].)

Next we show an interesting topological connection between a surface and the hom space of its quadrangulation from which the proof of Theorem 1.3 follows easily.

Let $G$ be a quadrangulation of a surface $S$. Let $\alpha$ be a map that maps the vertex $v_{e}$ of $M(G)$ to the class ${ }^{2}$ of $\{x\} \uplus\{y\}$ in $H(G) / \sigma$ where $e=x y$. Now $\alpha$ naturally extends to the edges of

\footnotetext{
${ }^{1}$ We use the notation $A \uplus B$ for the ordered pair containing $A$ and $B$ as it is customary in this context, cf. [9]. Alternatively, one can view $A \uplus B$ as the set $(A \times\{0\}) \cup(B \times\{1\}) \subseteq V(G) \times\{0,1\}$.

${ }^{2}$ Here we view $H(G) / \sigma$ as the quotient space obtained by identifying $p$ and $\sigma(p)$ for each point $p$ in the cell
} 
$M(G)$ (mapping them to 1-cells connecting the corresponding vertices) and also to the faces of $M(G)$ as follows. The image of the cycle face of $M(G)$ corresponding to the face $x y z t$ of $G$ will be the class of the cell $\{x, z\} \uplus\{y, t\}$. The image of the star face containing the vertex $v$ of $G$ is inside the class of the cell $\{v\} \uplus N(v)$. This makes $\alpha$ a continuous map $\alpha: S \rightarrow H(G) / \sigma$.

The map $\alpha$ lifts to a map $\beta: T \rightarrow H(G)$ where $T$ is a double cover of $S$. (See [8] for more details about covering spaces of graphs and surfaces.) Let us observe that the double cover restricted to the graph $G$ of the quadrangulation is isomorphic to the categorical product $G \times K_{2}$ (sometimes called the Kronecker product or the direct product). Let us consider the involution on $T$ interchanging the pairs of points with the same image in $S$. This makes $T$ into a $\mathbb{Z}_{2}$-space and $\beta$ a $\mathbb{Z}_{2}$-map. Notice that $T$ is the union of two disjoint copies of $S$ if and only if $G$ is bipartite.

Remark 1. Notice that the parity of the length of a cycle of a quadrangulation of a surface is determined by the homotopy type of the cycle as a surface cycle. These parities for the different homotopy types determine the double cover $T$ as follows: an even cycle on $S$ lifts to two cycles on $T$, while an odd cycle on $S$ lifts to a single cycle on $T$.

If we assume that all 4-cycles in $G$ are faces of the quadrangulation and $G$ is not a complete bipartite graph $K_{2, i}(i \leq 3)$, then $H(G)$ and $T$ are homotopy equivalent, with $\beta$ providing one direction of the homotopy equivalence. The exceptional graphs $K_{2, i}$ for $i \geq 1$ quadrangulate the sphere and for $i \leq 3$ all 4 -cycles are faces. To see the homotopy equivalence consider the maximal cells of $H(G)$ - these correspond to maximal complete bipartite subgraphs of $G$, which are the face cycles and the stars of vertices. It is not hard to see that $G$ has no vertex of degree less than 3. If $G$ is 3-regular, $T$ and $H(G)$ are actually homeomorphic and $\beta$ can be chosen to be a homeomorphism. Two $(d-1)$-dimensional simplicial cells of $H(G)$ correspond to the star of a degree $d$ vertex of $G$, so if $G$ is not 3-regular, $T$ and $H(G)$ are not homeomorphic. It is easy to see though that only the edges of a $d$-cycle on the boundary of these cells appear in other cells, therefore the higher dimensional cell can be collapsed to a $d$-gon without changing the homotopy type. After this collapse of every higher dimensional cell we obtain a complex homeomorphic to $T$.

Proof of Theorem 1.3: The upper bound 4 follows from $\psi(G) \leq \chi(G)$ and from the easy part of Youngs' theorem (that every quadrangulation of the projective plane is 4-colorable). The nontrivial part of the result is establishing that if $G$ is not bipartite it must have no local 3-coloring.

Consider the map $\beta: T \rightarrow H(G)$ constructed above. Here $T$ is the double cover of the projective plane and it is not the union of two disjoint copies of that plane as $G$ is not bipartite. The only possibility left for $T$ is therefore the sphere, $T=\mathbb{S}^{2}$. The existence of a $\mathbb{Z}_{2}$-map $\beta: \mathbb{S}^{2} \rightarrow H(G)$ implies that $\psi(G) \geq 4$, as proved in [18].

Remark 2: Among the graphs investigated in [17, 18] 4-chromatic generalized Mycielski graphs are known to quadrangulate the projective plane, see [6], while deleting some edges from 4-chromatic Schrijver graphs one obtains quadrangulations of the Klein bottle. In fact, it was the investigation of the local chromatic number of the latter graphs that led us to the proof presented in the next section and to consider the local chromatic number of surface quadrangulations in general.

complex $\mathrm{H}(\mathrm{G})$. 


\section{Semi-free groups}

In this section we present an intermediate step toward Theorem 1.4(i). This algebraic approach proves the statement for the non-orientable surfaces of genus one or two, i.e., for the projective plane and the Klein bottle.

Let $H$ be a graph. The semi-free group $\Gamma_{H}$ is the following group given by generators and relations. The generators are the vertices of $H$ while the relations are $x y=y x$ for the edges $x y$ of $H$. If $E(H)=\emptyset$ we obtain the free group, for a complete graph we obtain a free Abelian group. Thus, one can consider semi-free groups as a common generalization of free and free Abelian groups.

Let $G$ be a quadrangulation of a surface $S$ and let $c$ be a local 3-coloring of $G$. Our goal is to prove a lower bound on the genus of $S$ if the quadrangulation is odd.

Let $m$ be the number of colors used by the coloring $c$ and assume that these are the elements of $[m]=\{1, \ldots, m\}$. Consider the Kneser graph $H=\mathrm{K} G(m, 2)$ and the semi-free group $\Gamma=\Gamma_{H}$. The generators of $\Gamma$ are the vertices of $H$, which are the 2-element subsets of $[m]$. (Recall that two such vertices are adjacent in $\mathrm{K} G(m, 2)$ iff they represent disjoint subsets.) For $i, j \in[m]$ we introduce the notation

$$
x_{i, j}= \begin{cases}\varepsilon & \text { if } i=j \\ \{i, j\} & \text { if } i<j \\ \{i, j\}^{-1} & \text { if } j<i .\end{cases}
$$

where $\varepsilon$ is the identity element of $\Gamma$ and $\{i, j\}$ is a generator of $\Gamma$ if $i \neq j$. These group elements clearly satisfy $x_{i, j}=x_{j, i}^{-1}$ for any $i, j \in[m]$ and by the definition of the Kneser graph we have $x_{i, j} x_{k, l}=x_{k, l} x_{i, j}$ whenever $\{i, j\}$ and $\{k, l\}$ are disjoint. Moreover, we have

$$
x_{i, j} x_{j, k}=x_{i, k} \text { whenever }|\{i, j, k\}| \leq 2 .
$$

Consider the medial graph $M(G)$ of $G$. (See the definition in Section 2.) We consider the edges of $M(G)$ as oriented edges with both orientations of each (unoriented) edge being present. We label these oriented edges by elements of the group $\Gamma$. Consider the oriented edge $w=\left(v_{e}, v_{f}\right)$, where $e$ and $f$ are edges of $G$. Notice that $e=a b$ and $f=a d$ must be adjacent edges. In this case we label the oriented edge $w$ by $l(w)=x_{c(b), c(d)} \in \Gamma$. We let the label $l(W)$ of a walk $W$ on $M(G)$ be the product of the labels along the walk.

Lemma 3.1 For the opposite orientations $w$ and $w^{\prime}$ of the same edge we have $l\left(w^{\prime}\right)=l(w)^{-1}$. For a walk $W$ around a face of $M(G)$ we have $l(W)=\varepsilon$.

Proof. The first statement is trivial. A walk $W$ around a cycle face of $M(G)$ consists of four edges. See Figure 1 for this case. Let the vertices around the face of $G$ containing $W$ be $a_{0}, a_{1}, a_{2}, a_{3}$, connected by the edges $e_{i}=a_{i} a_{i+1}$ (where all indices are taken modulo 4 ). The walk $W$ consists of the oriented edges $w_{i}=\left(e_{i}, e_{i+1}\right) \quad(0 \leq i \leq 3)$ and we have $l\left(w_{i}\right)=x_{c\left(a_{i}\right), c\left(a_{i+2}\right)}$. Here $l\left(w_{1}\right)=x_{c\left(a_{1}\right), c\left(a_{3}\right)}=x_{c\left(a_{3}\right), c\left(a_{1}\right)}^{-1}=l\left(w_{3}\right)^{-1}$. Similarly, we have $l\left(w_{0}\right)=l\left(w_{2}\right)^{-1}$. The labels $l\left(w_{0}\right)=x_{c\left(a_{0}\right), c\left(a_{2}\right)}$ and $l\left(w_{1}\right)=x_{c\left(a_{1}\right), c\left(a_{3}\right)}$ commute since if the sets $\left\{c\left(a_{0}\right), c\left(a_{2}\right)\right\}$ and $\left\{c\left(a_{1}\right), c\left(a_{3}\right)\right\}$ were not disjoint, $c$ would not be a proper coloring. Consequently, $l(W)=\varepsilon$.

Let us now consider the walk $W$ around a star face of $M(G)$ as on Figure 2. Let $a$ be the vertex of $G$ inside this face and $b_{1}, \ldots, b_{d}$ the neighbors of $a$ in this order. We have

$$
l(W)=x_{c\left(b_{1}\right), c\left(b_{2}\right)} x_{c\left(b_{2}\right), c\left(b_{3}\right)} \ldots x_{c\left(b_{d-1}\right), c\left(b_{d}\right)} x_{c\left(b_{d}\right), c\left(b_{1}\right)} .
$$




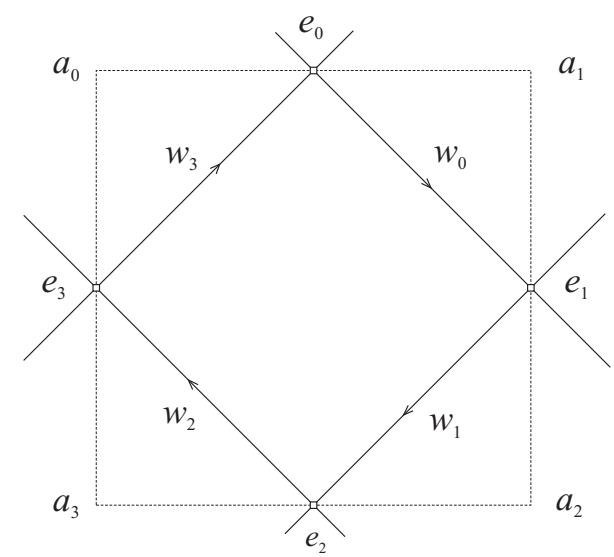

Figure 1: A cycle face in $M(G)$

As $c$ is a local 3-coloring there are at most 2 distinct ones among the colors $c\left(b_{i}\right)$. So Equation $(1)$ applies and simplifies the above expression to $l(W)=x_{c\left(b_{1}\right), c\left(b_{1}\right)}=\varepsilon$.

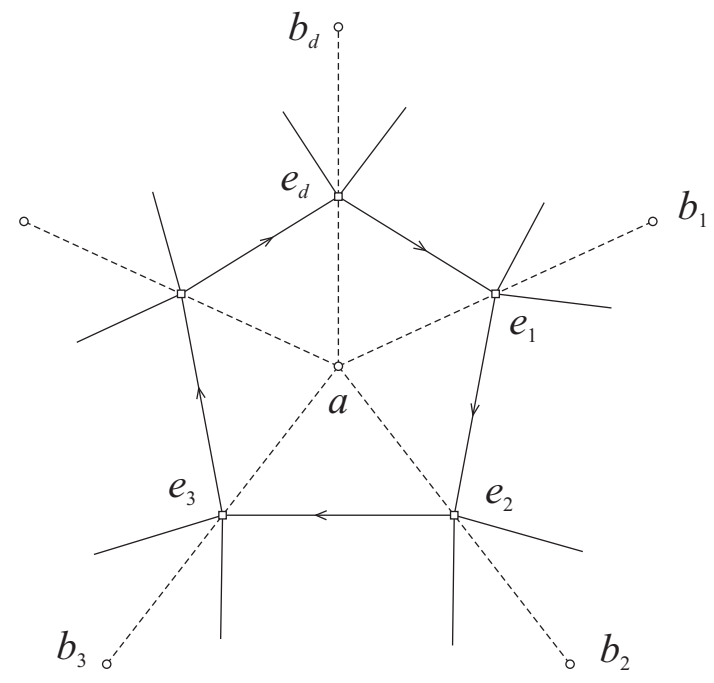

Figure 2: A star face in $M(G)$

Let us fix a vertex $v_{e_{0}}$ of $M(G)$ as the base point of $S$. If two closed walks on $M(G)$ starting at the base point are homotopic on $S$, then one can be transformed to the other by a sequence of steps, where each step is the introduction or removal of a closed sub-walk which is either a facial walk or an edge traversed both ways. By our last lemma these steps do not alter the label of the walk, so the label is determined by the homotopy type. As any homotopy type can be represented by a closed walk on $M(G)$, we have a map $\alpha: \pi_{1}(S) \rightarrow \Gamma$ from the fundamental group $\pi_{1}(S)$ of $S$ that gives as its value to each element of the fundamental group the label of the walks in the corresponding homotopy class. Clearly, this is a homomorphism.

We shall need another property of products in the semi-free group $\Gamma_{\mathrm{K} G(m, 2)}$. 
Lemma 3.2 Let $c_{1}, \ldots, c_{t}$ be colors in $[m]$, where $c_{i+1} \neq c_{i}$ for $i=1, \ldots, t-1$, and $c_{1} \neq c_{t}$. Let $\alpha=x_{c_{t}, c_{2}} x_{c_{1}, c_{3}} \ldots x_{c_{t-2}, c_{t}} x_{c_{t-1}, c_{1}}$. If $t$ is odd, then $\alpha \neq \varepsilon$.

Proof. Let us assume the lemma is false and consider a counterexample where a minimum number among the factors $X_{i}=x_{c_{i-1}, c_{i+1}}$ are non-identity. (Here and in the rest of the proof, all indices are understood modulo $t$.) There must be such non-identity factors as otherwise we would have $c_{i-1}=c_{i+1}$ for all $i$ and therefore (as $t$ is odd) that all the $c_{i}$ are the same.

Note that each factor $X_{i}$ is the identity or a generator or the inverse of a generator in the semi-free group $\Gamma$. We use the observation of Baudisch [2] that if a nontrivial product of generators and their inverses in a semi-free group is the identity, then one finds a generator and its inverse in the product such that they commute with all factors separating them (so they cancel each other). We may therefore choose indices $1 \leq i<j \leq t$ with $X_{i} \neq \varepsilon, X_{j}=X_{i}^{-1}$ and $X_{i}$ commuting with all $X_{s}$ for $i<s<j$. We choose $j$ so that $X_{j}$ is the first occurrence of $X_{i}^{-1}$ after the factor $X_{i}$. We have $c_{s}=c_{i+1}$ for all $i<s<j$ with $s-i$ odd, because otherwise for the smallest $s$ breaking this rule either $X_{s-1}$ would not commute with $X_{i}$ or $X_{s-1}=X_{i}^{-1}$ would hold contradicting the minimality of $j$. For $i<s<j$ with $s-i$ even we have $c_{s} \neq c_{i+1}$, because adjacent colors must differ. This implies that $j-i$ is even as $c_{j-1}=c_{i+1}$. Furthermore, $c_{s} \neq c_{i-1}$ for $i<s<j$ as otherwise $X_{s-1}$ and $X_{i}$ would not commute for the first such $s$.

Let us modify now the colors $c_{s}$ for $i<s<j, s-i$ odd to $c_{s}^{\prime}=c_{i-1}$ without changing the other colors. This does not create equal neighboring colors and changes only two factors: $X_{i}^{\prime}=X_{j}^{\prime}=\varepsilon$. We obtain another counterexample to the claim this way, and this counterexample has a smaller number of non-identity factors. This contradicts our choice and by this contradiction proves the lemma.

Lemma 3.3 If $\alpha(y)=\varepsilon$ for some class $y$ in the fundamental group of $S$, then any walk on $G$ representing $y$ has even length.

Proof. Assume for contradiction that $\alpha(y)=\varepsilon$ but the length $t$ of a closed walk $W$ representing $y$ is odd. Note that here we have the non-standard view of the walk $W$ as "starting" at the base point $v_{e_{0}}$ that happens to be in the middle of the edge $e_{0}$ of $G$. However, this means that the walk $W$ uses at the beginning one half of the edge $e_{0}$, and traverses the other half when coming back at the end. To apply the definition of $\alpha$, we have to find first a walk $W^{\prime}$ on $M(G)$ homotopic to $W$. Let $a_{1}, \ldots, a_{t}$ be the vertices of $G$ along $W$. Since $W$ starts and ends at the base point, we have $e_{0}=a_{1} a_{t}$. Let $e_{i}$ be the edge of $G$ connecting $a_{i}$ and $a_{i+1}$ for $1 \leq i<t$. We construct the walk $W^{\prime}$ on $M(G)$ as follows. It starts at the base point $v_{e_{0}}$ and then it passes through all the points $v_{e_{i}}$ for $1 \leq i<t$ in order before returning to the base point. We let the part $W_{i}$ of $W^{\prime}$ between $v_{e_{i-1}}$ and $v_{e_{i}}$ follow the boundary of the star face of $M(G)$ containing $a_{i}$ for $1 \leq i \leq t$. The indices here and in the rest of this proof are understood modulo $t$.

Let $c_{i}=c\left(a_{i}\right)$. As in the proof of Lemma 3.1 we can use the fact that $c$ is a local 3-coloring and Equation (1) to conclude that $l\left(W_{i}\right)=x_{c_{i-1}, c_{i+1}}$. We thus have

$$
\alpha(y)=l\left(W^{\prime}\right)=\prod_{i=1}^{t} l\left(W_{i}\right)=x_{c_{t}, c_{2}} x_{c_{1}, c_{3}} \ldots x_{c_{t-2}, c_{t}} x_{c_{t-1}, c_{1}} .
$$

Lemma 3.2 shows that a product of this form with $t$ odd is never the identity if $c_{i} \neq c_{i+1}$ for all $1 \leq i \leq t$. Note that neighboring colors $c_{i}$ and $c_{i+1}$ are distinct in our case as $c$ is a proper coloring, so this completes the proof. 
Remark 3. One can try to simplify the argument presented in this section by letting all pairs of generators commute in $\Gamma$, i.e., considering a commutative factor of $\Gamma$. The argument breaks down because Lemma 3.3 does not hold in this case. Assume $G$ has a cycle of length 9 with the vertices along the cycle colored $2,1,2,3,1,3,4,1,4$. Lemma 3.3 applies and $\alpha(y) \neq \varepsilon$ in $\Gamma$ for the class $y$ containing this cycle. But $\alpha(y)$ vanishes in any commutative factor of $\Gamma$.

Theorem 3.4 Let $g$ be a positive integer and assume the equality $z_{1}^{2} z_{2}^{2} \ldots z_{g}^{2}=\varepsilon$ implies $z_{1} z_{2} \ldots z_{g}=\varepsilon$ in the semi-free group $\Gamma_{\mathrm{K} G(m, 2)}$, where, as before, $\varepsilon$ denotes the identity element of the group. Then no odd quadrangulation of a non-orientable surface of genus $g$ has a local 3-coloring using at most $m$ colors.

Proof. Let $S$ be the non-orientable surface of genus $g$. The fundamental group $\pi_{1}(S)$ can be presented as $<y_{1}, \ldots, y_{g} \mid y_{1}^{2} y_{2}^{2} \ldots y_{g}^{2}=1>$. (Cf., e.g., [8] for more details about fundamental groups of surfaces.) Assume $S$ has a quadrangulation $G$ with a local 3-coloring using $m$ colors. We need to prove that this quadrangulation is even. With the homomorphism $\alpha: \pi_{1}(S) \rightarrow$ $\Gamma_{\mathrm{K} G(m, 2)}$ defined above we let $z_{i}=\alpha\left(y_{i}\right)$ for $1 \leq i \leq g$. We clearly have $z_{1}^{2} z_{2}^{2} \ldots z_{g}^{2}=\varepsilon$. By our assumption this implies $z_{1} z_{2} \ldots z_{g}=\varepsilon$, so $\alpha(y)=\varepsilon$ for $y=y_{1} y_{2} \ldots y_{g}$.

The surface $S$ can be obtained by placing $g$ crosscaps on the sphere and $y_{i}$ can be chosen to be the class of a loop going only through the $i$ th crosscap, so $y$ will be the class of a loop going through each crosscap once.

If we cut $S$ along a simple cycle in the homotopy class $y$ we obtain an orientable surface. If $y$ can be represented as a cycle $C$ of $G$, then one can cut $S$ along $C$ and consistently orient the obtained surface and in it all the faces of the quadrangulation $G$. With this orientation exactly the edges of $C$ break the consistency of the orientation and thus the parity of the quadrangulation $G$ is the same as the parity of the length of the cycle $C$.

In general we cannot assume the existence of a simple cycle in $G$ representing the class $y$ but we can always represent $y$ by a closed walk $W$ on $G$. We claim that the length of the walk has the same parity as the quadrangulation $G$. To see this one can refine the quadrangulation without changing its parity or that of any cycle till one finds a simple cycle homotopic to $W$ or alternatively one can prove that there is an orientation of the faces of $G$ where the consistency is broken at exactly the edges traversed an odd number of times by $W$.

By Lemma 3.3, the length of $W$ must be even because $\alpha(y)=\varepsilon$. This shows that $G$ is an even quadrangulation and completes the proof of the theorem.

We proved Theorem 3.4 to find another proof for Theorem 1.3 that extends also to higher genus surfaces.

The non-existence of an odd, locally 3-colorable quadrangulation of the projective plane follows now from the fact that $x^{2}=\varepsilon$ implies $x=\varepsilon$ in every semi-free group. This statement, and more generally that semi-free groups have no torsion elements, was proved by Baudisch [2].

In another paper [3], Baudisch proved that any two non-commuting elements of a semi-free group freely generate a free group of rank 2 . Thus $x^{2} y^{2}=\varepsilon$ implies that $x$ and $y$ commute. So we have $(x y)^{2}=x^{2} y^{2}=\varepsilon$ and by the earlier result $x y=\varepsilon$. This establishes that the Klein bottle (the non-orientable surface of genus 2 ) has no odd, locally 3-colorable quadrangulations.

To apply Theorem 3.4 to the next surface we would need that $x^{2} y^{2} z^{2}=\varepsilon$ implies $x y z=\varepsilon$ in semi-free groups, but we were not able to prove this. 
We can turn Theorem 3.4 around and use its counterpositive form. From the odd quadrangulation claimed in Theorem 1.4(ii) we conclude that there are elements $z_{1}, z_{2}, z_{3}, z_{4}$ and $z_{5}$ in the semi-free group $\Gamma_{\mathrm{K} G(6,2)}$ satisfying $z_{1}^{2} z_{2}^{2} z_{3}^{2} z_{4}^{2} z_{5}^{2}=\varepsilon$ and $z_{1} z_{2} z_{3} z_{4} z_{5} \neq \varepsilon$. The construction in Section 5 gives rise to a description of these elements, see Table 1 . We found these elements by "reverse engineering". First, we traced out standard generators of the fundamental group (certain cycles that bound Möbius strips) and calculated the elements of the semi-free group they are mapped to by the homomorphism $\alpha$. Finally, we played around with those group elements to make their presentation shorter. Note that as a result of the properties of the construction our example uses only nine of the fifteen generators of $\Gamma_{\mathrm{K} G(6,2)}$, so our example actually lives in the semi-free group $\Gamma_{C_{3}^{2}}$, where $C_{3}^{2}$ is the Cartesian square of the 3 -cycle.

$$
\begin{aligned}
z_{1} & =a_{25} a_{14} a_{24}^{-1} a_{15}^{-1} \\
z_{2} & =a_{15} a_{24} a_{14}^{-1} a_{16} a_{36}^{-1} a_{15}^{-1} a_{35} a_{14} a_{24}^{-1} a_{15}^{-1} \\
z_{3} & =a_{15} a_{24} a_{35}^{-1} a_{36} a_{14}^{-1} a_{26}^{-1} \\
z_{4} & =a_{26} a_{14} a_{36}^{-1} a_{24}^{-1} a_{34} a_{14}^{-1} \\
z_{5} & =a_{14} a_{34}^{-1} a_{36} a_{24} a_{16}^{-1} a_{15} a_{25}^{-1} a_{14}^{-1} \\
z_{1}^{2} z_{2}^{2} z_{3}^{2} z_{4}^{2} z_{5}^{2} & =\varepsilon \\
z_{1} z_{2} z_{3} z_{4} z_{5} & =a_{25} a_{16} a_{15}^{-1} a_{14} a_{16}^{-1} a_{15} a_{25}^{-1} a_{14}^{-1} \neq \varepsilon
\end{aligned}
$$

Table 1. Five elements in the semi-free group $\Gamma_{K G(6,2)}$ showing peculiar behavior.

The generator corresponding to the vertex $\{i, j\}$ of $\mathrm{K} G(6,2)$ is denoted by $a_{i j}$.

From the quadrangulation, whose existence is claimed in Theorem 1.5(ii), one can construct a similar list of 7 elements in the semi-free group corresponding to $K G(5,2)$, that is, the Petersen graph. See Table 2.

$$
\begin{aligned}
w_{1} & =b_{23} b_{13}^{-1} b_{24}^{-1} b_{14} \\
w_{2} & =b_{14}^{-1} b_{13} b_{24} b_{35} b_{25}^{-1} \\
w_{3} & =b_{25} b_{24}^{-1} b_{35}^{-1} b_{34} \\
w_{4} & =b_{34}^{-1} b_{35} b_{24} b_{34}^{-1} b_{13}^{-1} b_{35}^{-1} b_{15} b_{23}^{-1} b_{35}^{-1} b_{34} \\
w_{5} & =b_{34}^{-1} b_{35} b_{15}^{-1} b_{23} b_{12} b_{34} \\
w_{6} & =b_{34}^{-1} b_{12}^{-1} b_{23}^{-1} b_{15} b_{45}^{-1} \\
w_{7} & =b_{23} b_{45} a_{15}^{-1} b_{34} b_{13} b_{23}^{-1} \\
w_{1}^{2} w_{2}^{2} w_{3}^{2} w_{4}^{2} w_{5}^{2} w_{6}^{2} w_{7}^{2} & =\varepsilon \\
w_{1} w_{2} w_{3} w_{4} w_{5} w_{6} w_{7} & =b_{23} b_{35} b_{34}^{-1} b_{13}^{-1} b_{35}^{-1} b_{34} b_{13} b_{23}^{-1} \neq \varepsilon
\end{aligned}
$$

Table 2. Seven elements in the semi-free group $\Gamma_{\mathrm{K} G(5,2)}$ showing peculiar behavior. The generator corresponding to the vertex $\{i, j\}$ of $\operatorname{K} G(5,2)$ is denoted by $b_{i j}$. 


\section{A combinatorial approach}

In this section we present the most elementary and, at the same time, at least by our current knowledge, the most effective approach toward proving that certain quadrangulations have no local 3-colorings. It is based on examining properties of minimal counterexamples.

Let us call a triple $(G, S, c)$ a suitable quadrangulation, if $G$ is an odd quadrangulation of the (non-orientable) surface $S$ and $c$ is a local 3-coloring of $G$. A suitable quadrangulation $(G, S, c)$ is called a minimal quadrangulation if no surface of genus less than that of $S$ has a suitable quadrangulation and $S$ has no suitable quadrangulation with fewer faces.

Lemma 4.1 If $(G, S, c)$ is a minimal quadrangulation and $F$ is a face of this quadrangulation, then $F$ has four distinct vertices. Moreover, these vertices receive two or four distinct colors in the local 3-coloring. The neighbors of any vertex in $G$ receive exactly two distinct colors.

Proof. As $c$ is a proper coloring, we have no loops, so only diagonally opposite vertices of $F$ can coincide. Let us assume that the same vertex $x$ appears at both endpoints of the diagonal $d$ of the face $F$. Then the two edges of $F$ connecting $x$ to another vertex of $F$ are parallel edges or they coincide. Let us cut the face $F$ out from $S$ and close $S$ up by identifying these pairs of parallel edges of $F$ or by removing the edge if the two neighboring sides of $F$ coincided. Let $S^{\prime}$ be the space obtained from $S$ this way and $G^{\prime}$ be the resulting graph on $S^{\prime}$. As we only identified parallel edges, $c$ is a local 3-coloring of $G^{\prime}$. Considering any orientation of the faces of $G$ and the same orientation of the faces of $G^{\prime}$ one sees that an edge of $G^{\prime}$ obtained by identification breaks consistency of this orientation if and only if exactly one of the corresponding two edges in $G$ does so, other edges of $G^{\prime}$ break consistency of the orientation in $G^{\prime}$ if and only if they do so in $G$, while edges removed from $G \operatorname{did}$ not break consistency there. Therefore, since $G$ is an odd quadrangulation, $G^{\prime}$ must be odd, too. Note, however, that $S^{\prime}$ is not necessarily a surface as the neighborhood of $x$ can be strange (see below), so for the previous sentence to make sense we have to allow a somewhat extended definition of parity of quadrangulations.

In the following case analysis we always find a quadrangulation showing that $(G, S, c)$ is not minimal. This is done in slightly different ways depending on the topology of the diagonal $d$.

In the simplest case $d$ is a one-sided simple closed curve on $S$ (which is clearly non-separating). In this case $S^{\prime}$ is a surface. (To see this, it is enough to check how the faces incident to $x$ are arranged around this point.) So $\left(G^{\prime}, S^{\prime}, c\right)$ is a suitable quadrangulation of $S^{\prime}$. The surface $S^{\prime}$ is non-orientable as it has an odd quadrangulation. Its Euler characteristic is one more than that of $S$, as $G^{\prime}$ has one fewer face and two fewer edges, and it has the same number of vertices as $G$. So the genus of $S^{\prime}$ is one less than that of $S$ contradicting the minimality of $(G, S, c)$.

Our second case is when a pair of coinciding neighboring edges got removed from $G$. This makes $d$ a (trivial) separating cycle on $S, S^{\prime}$ a surface homeomorphic to $S$ and $\left(S^{\prime}, G^{\prime}, c\right)$ a suitable quadrangulation contradicting the minimality of $(S, G, c)$. We mention here that if two pairs of neighboring edges of $F$ got removed we would end up with $S^{\prime}$ empty, but this comes only from the path $P_{3}$ as an even quadrangulation of the 2 -sphere so it is is not possible.

Next we consider the case that $d$ is separating, but no edge of $F$ got removed. Now $S^{\prime}$ is the union of two surfaces having only $x$ as their common point. The graph $G^{\prime}$ quadrangulates both surfaces. As the total number of edges breaking consistency of orientation in $G^{\prime}$ is odd, the subgraph $G^{\prime \prime}$ of $G^{\prime}$ quadrangulating one of these surfaces $S^{\prime \prime}$ is an odd quadrangulation. Clearly, 
$S^{\prime \prime}$ is a non-orientable surface that has an odd quadrangulation $G^{\prime \prime}$ and $G^{\prime \prime}$ has fewer faces than $G$ but it also inherits a local 3-coloring. As $(G, S, c)$ is a minimal quadrangulation, the genus of $S^{\prime \prime}$ must be strictly larger than that of $S$. But this is impossible as the sum of the Euler characteristics of the two surfaces $S^{\prime}$ consists of is exactly 2 more than the Euler characteristic of $S$, and the other surface in $S^{\prime}$ can contribute at most 2 to the sum.

Our remaining last case is when the diagonal $d$ is a non-separating 2 -sided cycle on $S$. Here $S^{\prime}$ is a "pinched surface", it can be made into a surface by replacing $x$ with two points. Let $S^{\prime \prime}$ be the surface so obtained and let $G^{\prime \prime}$ be the quadrangulation of $S^{\prime \prime}$ obtained in this process. The Euler characteristic of $S^{\prime \prime}$ is two more than that of $S$ and its quadrangulation $G^{\prime \prime}$ is odd and has a local 3-coloring, thus $(G, S, c)$ is not minimal in this case either. This completes our proof that $F$ must have four distinct vertices.

Let the vertices along the facial cycle around $F$ be $x, y, z$ and $t$. As $c$ is a proper coloring, only the colors of $x$ and $z$, or the colors of $y$ and $t$ can coincide. In order to prove that these vertices cannot have exactly 3 distinct colors assume for a contradiction that $c(x)=c(z)$ and $c(y) \neq c(t)$. We do as above: we cut $F$ out from $S$ and close $S$ up by identifying $x$ with $z$ and also the edge $x y$ with the edge $z y$ and the edge $x t$ with $z t$. This time we obtain a surface $S^{\prime}$ homeomorphic to $S$. We also obtain a quadrangulation $G^{\prime}$ of $S^{\prime}$ with one fewer faces and just as above, it must be an odd quadrangulation. As we identified vertices with equal color, the graph $G^{\prime}$ inherits a proper coloring from $c$. We claim it is a local 3-coloring. This is because both $x$ and $z$ had $y$ and $t$ in their neighborhoods, so both neighborhoods must contain only vertices of color $c(y)$ and $c(t)$. Therefore this is also true for the vertex obtained by identifying $x$ and $z$. The contradiction with the minimality of $G$ shows that all faces of $G$ must have two or four distinct colors at their vertices.

Finally, we have to derive a contradiction from the assumption that the neighborhood of a vertex $x$ in $G$ is monochromatic. Let $F$ be a face incident to $x$, let $x, y, z$ and $t$ be the vertices along its facial walk. As $y$ and $t$ are neighbors of $x$ we must have $c(y)=c(t)$. By the earlier part of this lemma this implies that $F$ must have only two colors, so $c(x)=c(z)$. We apply the same procedure again: cut $F$ out from $S$, and close $S$ up by identifying the edge $x y$ with $z y$ and the edge $x t$ with $z t$. We obtain a quadrangulation $G^{\prime}$ of a surface $S^{\prime}$ with one fewer faces than in $G$. As before, $G^{\prime}$ is an odd quadrangulation, $S^{\prime}$ is homeomorphic with $S$, and $c$ gives rise to a local 3-coloring of $G^{\prime}$ as all neighbors of the common image of $x$ and $z$ have colors that appear in the $G$-neighborhood of $z$. The contradiction with the minimality of $(G, S, c)$ completes the proof of the lemma.

We call a face of a minimal quadrangulation bichromatic or four-chromatic depending on the number of distinct colors its vertices receive.

Lemma 4.2 Two four-chromatic faces of a minimal quadrangulation cannot share an edge.

Proof. Let $(G, S, c)$ be a minimal quadrangulation and let the vertices along the facial cycles of the faces on the edge $e=x y$ be $x, y, z, t$ and $x, y, z^{\prime}, t^{\prime}$. If both of these faces are four-chromatic, then $c(z)=c\left(z^{\prime}\right)$ as otherwise the vertex $y$ had three different colors in its neighborhood. Similarly, $c(t)=c\left(t^{\prime}\right)$. Let us obtain $G^{\prime}$ from $G$ by removing the edge $e$ and inserting a new edge connecting $z$ and $t^{\prime}$. Clearly, $G^{\prime}$ is also an odd quadrangulation of $S$ with just as many faces as $G$ and $c$ is a local 3-coloring of $G^{\prime}$. Thus $\left(G^{\prime}, S, c\right)$ is a minimal quadrangulation. Both faces 
incident to the new $z t^{\prime}$ edge have three distinct colors. This contradicts Lemma 4.1 and proves the present lemma.

We call a vertex of a minimal quadrangulation $(G, S, c)$ regular if its degree is 4 . The rest of the vertices of $G$ are called irregular. We define the auxiliary graph $H$ on the vertex set $V(H)=V(G)$ by connecting diagonally opposite vertices of the bichromatic faces of $G$. Note that in the auxiliary graph only vertices of equal color are connected.

Lemma 4.3 In a minimal quadrangulation each vertex has degree at least four. In the auxiliary graph regular vertices of the quadrangulation have degree 2 , irregular vertices have degree at least 3. In particular, vertices of degree 5 in $G$ have degree 3 in the auxiliary graph.

Proof. Let $x$ be a vertex of a minimal quadrangulation $(G, S, c)$. Its degree $d_{G}(x)$ in $G$ is the total number of faces incident to $x$, while its degree $d_{H}(x)$ in the auxiliary graph $H$ is the number of bichromatic faces incident to $x$. By Lemma 4.2 at least half of the faces incident to $x$ are bichromatic. Four-chromatic faces correspond to changes in color as we consider the neighbors of $x$ in their cyclic order. By Lemma 4.1 there must be such a change, and therefore at least two four-chromatic faces. The statements of this lemma follow.

Lemma 4.4 If a component $C$ of the auxiliary graph $H$ of a minimal quadrangulation $(G, S, c)$ is a cycle, then $G$ has at least two vertices of degree at least 8 that are adjacent in $G$ to a vertex of $C$.

Proof. Clearly, all vertices of $C$ are identically colored and by Lemma 4.3 they are all regular vertices.

A color is said to match a vertex $x$ of $C$ if it is the color $c(z)$ of a vertex $z$ that is diagonally opposite from $x$ in a four-chromatic face of $G$. Each vertex of $C$ is regular, so each is incident to exactly two four-chromatic faces, one on either side of $C$.

We claim that the same colors match every vertex of $C$. To see this it is enough to prove that the same colors match neighboring vertices along $C$. Let $x$ and $y$ be neighbors along $C$. As they are connected in $H$, they appear as diagonally opposite vertices of a bichromatic face $F_{1}$ of $G$. Consider a color $c(z)$ that matches $x$ with $z$ diagonally opposite from $x$ in the four-chromatic face $F_{2}$. As $x$ is a regular vertex the bichromatic and four-chromatic faces $F_{1}$ and $F_{2}$, both incident to $x$, must share an edge $x t$. Now ty is an edge of $F_{1}$ and as $y$ is a regular vertex, the other face $F_{3}$ incident to this edge must be four-chromatic. Let $u$ be the vertex diagonally opposite from $y$ in $F_{3}$. Clearly, $c(u)$ matches $y$. We finish the proof of the claim by observing that the neighborhood of $t$ in $G$ contains $x, z$ and $u$, so these vertices cannot have all distinct colors. Since $F_{2}$ and $F_{3}$ are four-chromatic faces, we have $c(z) \neq c(x)$ and $c(u) \neq c(y)=c(x)$, therefore we must have $c(z)=c(u)$.

Let us now fix a color $\alpha$ that matches the vertices of $C$ and consider the coloring $c^{\prime}$ of the vertices of $G$ given by $c^{\prime}(x)=\alpha$ for $x$ in $C$ and $c^{\prime}(x)=c(x)$ otherwise.

We claim that $c^{\prime}$ is a proper coloring of $G$. To see this it is enough to consider a vertex $x$ of $C$ and prove that no neighbor of $x$ in $G$ has color $\alpha$. As $\alpha$ matches $x$ we have $\alpha=c(z)$ with $z$ diagonally opposite from $x$ on a four-chromatic face $F$. The two other vertices on $F$ are neighbors of $x$ and have distinct colors neither of which is $\alpha$. As $c$ is a local 3-coloring all other neighbors of $x$ must also have one of these two colors, so none can have the color $\alpha$. 
It is easy to see that $c^{\prime}$ is not a local 3 -coloring. If it were, then $\left(G, S, c^{\prime}\right)$ would also be a minimal quadrangulation, but any face $F$ that has a vertex $x$ in $C$ and a vertex $z$ with $c(z)=\alpha$ as diagonally opposite vertices would have 3 distinct colors, contradicting Lemma 4.1.

We thus have a vertex $w$ that has at least 3 different colors under $c^{\prime}$ in its neighborhood. This is only possible if $w$ has some vertex $x$ of $C$ in its neighborhood and also some vertex $x^{\prime}$ of the same color $c(x)=c\left(x^{\prime}\right)$ but outside $C$. Consider the faces around $w$. We saw that four-chromatic faces correspond to alternations in the color of the neighbors of $w$, so there must be an even number of those faces. As vertices of $C$ are regular we see that there are two fourchromatic faces separated by a bichromatic face with two neighbors of $w$ on $C$. As we must also have $x^{\prime}$ among its neighbors this further means that four-chromatic faces incident to $w$ but not to its just mentioned two neighbors on $C$ must exist, so we have at least 4 four-chromatic faces around $w$. By Lemma 4.2 these faces must be separated by bichromatic faces, so the degree of $w$ is at least 8 .

To finish the proof of this lemma we have to establish that the vertex $w$ found above is not the only high degree vertex in the neighborhood of $C$. To see this, notice that $w$ has no neighbor $s$ with $c(s)=\alpha$ as otherwise it would have no more $c^{\prime}$-colors in its neighborhood than $c$-colors. But $w$ has a neighbor $x$ in $C$ and one of the two faces incident to the edge $x w$ is a four-chromatic face $F$. If $z$ is diagonally opposite from $x$ in $F$, then $\beta=c(z)$ matches $x$ and we can define the coloring $c^{\prime \prime}$ by re-coloring the vertices of $C$ to $\beta$. With this we find another high degree vertex as $w$, this one also in the neighborhood of $C$, but not having color $\beta$ in its neighborhood.

Proof of Theorem 1.4(i): We shall prove that for a minimal quadrangulation $(G, S, c)$ the genus $g$ of $S$ is at least 5 . The Euler characteristic of the non-orientable surface of genus $g$ is $2-g$. As $G$ is a quadrangulation, it has half as many faces as edges, so we have $2-g=$ $|V(G)|+|E(G)| / 2-|E(G)|$. Here $2|E(G)|=\sum d_{G}(x)$, where $d_{G}(x)$ is the degree of the vertex $x$ in $G$. We have

$$
\sum_{x \in V(G)}\left(d_{G}(x)-4\right)=4(g-2) .
$$

We call $d_{G}(x)-4$ the excess of the vertex $x$. Clearly, regular vertices have zero excess, irregular vertices have positive excess. Note that at this point (or rather at Lemma 4.3) we have re-proved Theorem 1.3, as for $g=1$ the total excess should be -4 .

We distribute the excess of irregular vertices to the colors. If a vertex has excess 1 (i.e., it is of degree 5), we give this excess to its color. If the excess of a vertex is 2 or 3 we give 2 of it to its color. If the excess of a vertex is at least 4 we still give 2 to its color and we give 1 to each of the two colors in its neighborhood. (Recall that any vertex has two colors in its neighborhood by Lemma 4.1.)

We have distributed not more, than the total excess of $4(g-2)$. We claim that each color that is used by the coloring $c$ receives at least 2 units of the overall excess. Indeed, the vertices of any color form one or more components of $H$. If such a component is a cycle, then by Lemma 4.4 the corresponding color receives 1 unit of excess from at least two distinct high degree neighboring vertices. Now consider a non-cycle component. By Lemma 4.3 each vertex in $H$ has degree at least 2 and degree 3 means that 1 unit of excess is given to the color of this vertex, while degree 4 or higher means that 2 units of excess is given to its color. As no (finite) component can have a single degree 3 vertex with all other vertices being of degree 2 , this proves the claim. 
By Theorem 1.1, $G$ is not colorable by 3 colors. This implies that $c$ uses at least 5 colors as any graph that has a local 3-coloring with 4 colors can, in fact, be properly colored with 3 colors (as we have observed after Theorem 1.4). So the total excess of $4(g-2)$ is at least $5 \cdot 2=10$, and we have $g \geq 5$ as claimed.

\section{Constructions}

In this section we construct several odd quadrangulations that are locally 3-colorable. In particular, we prove Theorems 1.4(ii) and 1.5(ii). Our starting points are the following graphs $U(m, r)$, defined in [4], characterizing local $r$-colorability.

Let $m \geq r$ be positive integers and $[m]=\{1, \ldots, m\}$. The vertex set $V(U(m, r))$ consists of the pairs $(i, A)$ with $i \in[m], i \notin A \subset[m]$ and $|A|=r-1$. The vertices $(i, A)$ and $(j, B)$ are adjacent in $U(m, r)$ if and only if $i \in B$ and $j \in A$. The natural coloring of $U(m, r)$ gives the color $i$ to each vertex $(i, A)$. This is a local $r$-coloring of $U(m, r)$.

By an elementary result proved in [4], a graph is locally $r$-colorable using at most $m$ colors if and only if it has a homomorphism to $U(m, r)$.

It will be beneficial to distinguish edges of $U(m, 3)$ appearing in triangles. These triangle edges connect $(i,\{j, k\})$ with $(j,\{i, k\})$ for some distinct colors $i, j, k \in[m]$.

We let $G_{0}$ be the subgraph obtained from $U(5,3)$ by removing all triangle edges. This is an edge-transitive graph on thirty vertices with sixty edges.

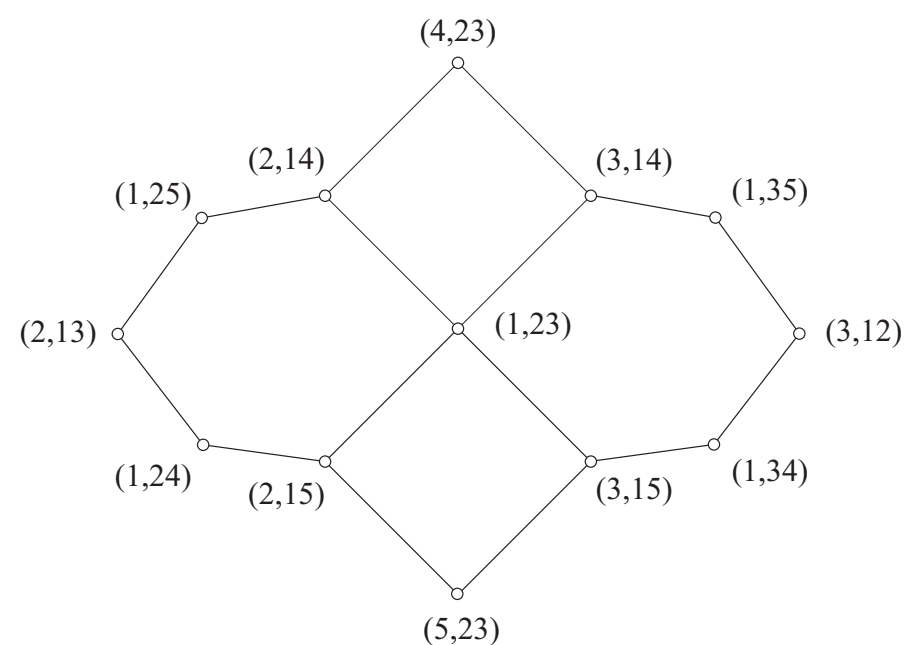

Figure 3: The faces around the vertex $(1,23)$ in $G_{0}$

We shall describe an embedding of $G_{0}$ into a surface by listing the faces. The embedding will have quadrilateral and hexagonal faces. We take all 4-cycles in $G_{0}$ as quadrilateral faces and we take the 6-cycles that receive only two distinct colors at the natural coloring as the hexagonal faces. Notice that each edge of $G_{0}$ appears in exactly one quadrilateral and exactly one hexagonal face. To check that these faces give rise to a surface, one has to check that the faces form a disk neighborhood around each vertex. By transitivity of $G_{0}$, it suffices to verify this for any vertex of $G_{0}$, and we refer to Figure 3 for details (where we use the notation $(i, j k)$ 
to denote the vertex $(i,\{j, k\}))$.

Altogether, we have fifteen quadrilateral faces and ten hexagonal faces. This makes the Euler characteristic of the resulting surface $S_{0}$ equal to $30-60+25=-5$. Therefore $S_{0}$ is the non-orientable surface of genus 7 .

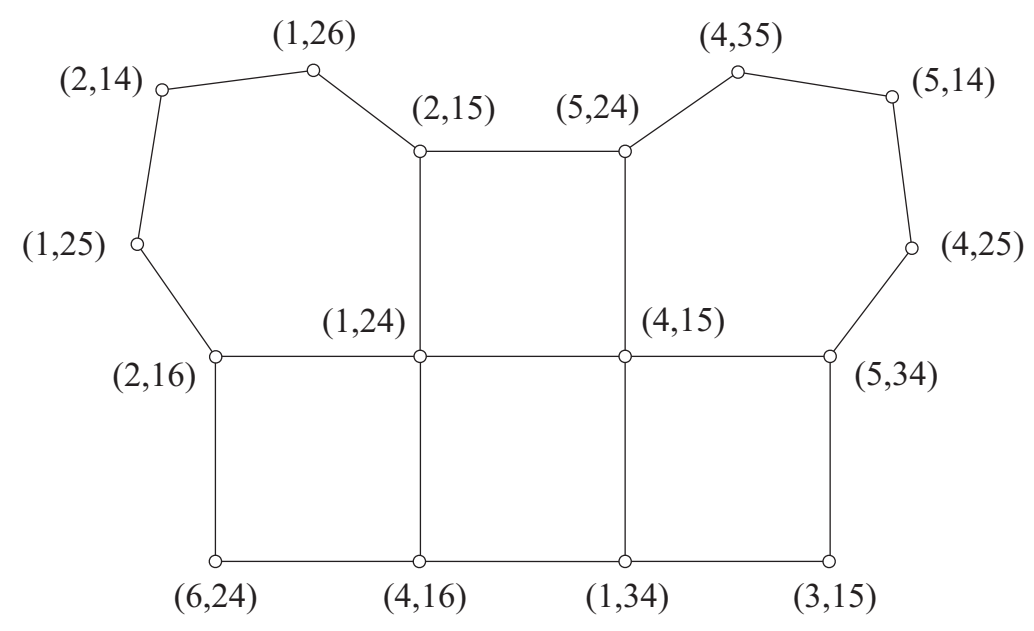

Figure 4: The faces around vertices $(1,24)$ and $(4,15)$ in $G_{1}$

To obtain a similar example on a surface of smaller genus, we start with the graph $U(6,3)$. Let $G_{1}$ be the subgraph of $U(6,3)$ consisting of the vertices $(i, H)$, with $|H \cap\{1,2,3\}|=1$ (and hence also $|H \cap\{4,5,6\}|=1$ ), and with all non-triangle edges connecting these vertices. This is a vertex-transitive graph on 36 vertices with 72 edges.

We embed $G_{1}$ into a surface by listing the resulting faces. As in the case of $G_{0}$, we have quadrilateral and hexagonal faces; we take all 4-cycles of $G_{1}$ as quadrilateral faces and the 6cycles of $G_{1}$ that receive two colors at the natural coloring as the hexagonal faces. We have to check again that these faces form a disk neighborhood around each vertex, and they do (cf. Figure 4). So these faces form a surface $S_{1}$. We have 18 quadrilateral faces that receive four colors at the natural coloring and nine further quadrilateral faces receiving two colors, and six hexagonal faces. This makes the Euler characteristic of $S_{1}$ equal to $36-72+33=-3$. Therefore $S_{1}$ is the non-orientable surface of genus 5 .

To obtain quadrangulations of $S_{0}$ and $S_{1}$ from the above examples, we add a main diagonal to every hexagonal face of $G_{0}$ and $G_{1}$. The choice, which of the three main diagonals to add is arbitrary for each such face. It is simple to check that the resulting graphs $G_{0}^{\prime}$ and $G_{1}^{\prime}$ are odd quadrangulations of $S_{0}$ and $S_{1}$, respectively. Notice that the new diagonal edges of $G_{0}^{\prime}$ are still edges of the original graph $U(m, 3)(m=5$ or 6$)$ : they are triangle edges that were removed earlier. This ensures that the natural coloring locally 3-colors $G_{0}^{\prime}$ with 5 colors and locally 3 -colors $G_{1}^{\prime}$ with 6 colors.

We have just given constructions for the first cases of Theorems 1.4(ii) and 1.5(ii). To finish the proof of these results we need to give examples similar to $G_{0}^{\prime}$ and $G_{1}^{\prime}$ but quadrangulating higher genus surfaces. For this note that both quadrangulations $G_{0}^{\prime}$ and $G_{1}^{\prime}$ have pairs of faces sharing a common edge and receiving only two colors. Removing the common edge we get (back) a hexagonal face. We add a crosscap in the middle of this hexagonal face and quadrangulate 
the resulting surface by adding all three diagonals through the crosscap. The surface we obtain is of genus one higher than our original surface, the resulting quadrangulation is still odd, and the same coloring still locally 3 -colors the new graph. Note that the resulting graph will again have neighboring faces receiving only two colors, so this process can be repeated any number of times finishing the proof of the existence claims.

We remark that here we increase the genus of the surface with adding new edges but no new vertices to the quadrangulating graphs. This soon results in quadrangulating graphs with parallel edges. If one prefers quadrangulating graphs without parallel edges one can subdivide each edge into three edges and each face into a grid of nine faces. This has no effect on the parity, but it gets rid of any parallel edges. Any local 3-coloring extends to the subdivided graph using the same set of colors.

We also note that applying the above process exactly once to every hexagonal face of $G_{0}$ or $G_{1}$ we can see that the graph $U(5,3)$ and an induced subgraph of $U(6,3)$ are themselves odd quadrangulations of some surfaces: these are the non-orientable surfaces of genus 17 and 11, respectively.

\section{Local 3-colorings with five colors}

In this section we turn the construction proving Theorem 1.5(ii) around and use it to prove the impossibility result Theorem 1.5(i).

Let $G$ be a quadrangulation of a surface and assume each edge of $G$ is oriented. We call a face of $G$ odd if the edges around it are oriented with three in one cyclic direction and one in reverse. The following simple observation justifies this name.

Lemma 6.1 Let $G$ be a quadrangulation of a surface in which all edges are oriented. Then $G$ is an odd quadrangulation if and only if the number of odd faces is odd.

Proof. Orient the faces of $G$ in an arbitrary manner and consider the facial walks in this direction. The parity of all face-edge pairs with the facial walk traversing the edge in reverse direction is the parity of all faces contributing an odd number - these are the odd faces - and also it is the parity of all edges contributing an odd number - these are the orientation breaking edges.

Proof. (of Theorem 1.5(i)). We consider the graph $G_{0}$ described in Section 5 as the graph embedded in the genus 7 non-orientable surface $S_{0}$. Add all main diagonals in all hexagonal faces to obtain a drawing of $U(5,3)$ in $S_{0}$ where each of these newly added triangular edges cross two other triangular edges.

Let $G$ be an odd quadrangulation of another surface $S$ and $c$ a local 3-coloring of $G$ with the five colors $\{1,2,3,4,5\}$. As we have mentioned in the previous section (and as proved in [4]) $c$ gives rise to a graph-homomorphism $f: G \rightarrow U(5,3)$ such that the natural coloring assigns the color $c(x)$ to $f(x)$ for every vertex $x$ of $G$. Let $\bar{f}: S \rightarrow S_{0}$ be a continuous extension of $f$. First extend $f$ from vertices to the points along the edges, then realize that the facial walk of any face of $G$ is mapped trivially to one or two edges of $U(5,3)$ or within a face of $G_{0}$ and thus can easily be extended within the same face. 
We want to use a simple result that relates the genera of two surfaces and the degree ${ }^{3}$ of a mapping between them. As the degree is defined for oriented surfaces we consider the orientable double covers $\tilde{S}_{0}$ of $S_{0}$ and $\tilde{S}$ of $S$ with an arbitrary orientation. Note that the Euler characteristic of the double cover is twice the Euler characteristic of the base, so the genera of $S$ and $\tilde{S}$ agree and so do the genera of $S_{0}$ and $\tilde{S}_{0}$. The map $\bar{f}: S \rightarrow S_{0}$ lifts to $\tilde{f}: \tilde{S} \rightarrow \tilde{S}_{0}$. Let $d$ be the degree of this map. Consider a quadrilateral face $F$ of $G_{0}$ and let $n_{F}$ be the number of faces of $G$ that $f$ maps onto $F$. Notice that the graphs $G$ and $G_{0}$ are also lifted to the orientable surfaces and the number of faces mapped to either one of the faces above $F$ is also $n_{F}$. To obtain the degree $d$ of the map $\tilde{f}$ one has to count these inverse images with appropriate signs depending on whether $\tilde{f}$ keeps the orientation of the face. In the notation from the footnote, we have $n_{F}=n_{F}^{+}+n_{F}^{-}$and $d=n_{F}^{+}-n_{F}^{-}$. Thus the parity of $d$ and $n_{F}$ agree. In particular, the parity of $n_{F}$ is independent of the choice of the quadrilateral face $F$. (Note that if using hexagonal faces of $G_{0}$, it would be harder to find the parity of the degree as some faces of $G$ map to parts of those hexagonal faces.) We use Kneser's formula [12], see also [20, p. 73]. It states that $g-1 \geq|d|\left(g_{0}-1\right)$ for the genera $g>0$ and $g_{0}$ of $\tilde{S}$ and $\tilde{S}_{0}$ (respectively) if a map of degree $d$ from $\tilde{S}$ to $\tilde{S}_{0}$ exists. We use the consequence of this formula that $d=0$ whenever $S$ has genus less than 7 .

We claim that $G$ is an odd quadrangulation if and only if the degree of $\tilde{f}$ is odd. As 0 is even, this claim finishes the proof of Theorem 1.5(i).

It is easier to show the claim using Lemma 6.1 considered as an alternative definition of odd quadrangulations.

To obtain an oriented version of $G$ let us orient an edge $x y$ from $x$ to $y$ if $c(x)<c(y)$. The odd faces will be exactly those with some colors $a<b<d<e$ around the face in this order. So $G$ is an odd quadrangulation if and only if $\sum n_{F}$ is odd, where the summation is for the quadrilateral faces $F$ of $G_{0}$ whose verices receive some colors $a<b<d<e$ in this order in the natural coloring. There are five such faces in $G_{0}$. This finishes the proof of the claim and with it the proof of Theorem 1.5(i).

Note that a proof similar to the above is not possible for Theorem 1.4(i) for two reasons. First, $G_{1}$ is too small a part of $U(6,3)$, there are large parts of $U(6,3)$ that are not represented by $G_{1}$. Second, even if $G_{1}$ was all (or a large part) of $U(6,3)$, this line of thought would only prove the impossibility of a local 3-coloring of an odd quadrangulation of small genus surfaces using 6 colors, whereas Theorem 1.4(i) states the same for any number of colors.

\section{$7 \quad$ Local 4-colorings of triangulations}

If $Q$ is a quadrangulation, we define the face subdivision $T(Q)$ of $Q$ as the triangulation of the same surface that is obtained from $Q$ by adding a vertex in each face and joining it to the four vertices on its boundary. The behavior of the local chromatic number exhibited in

\footnotetext{
${ }^{3}$ The degree of a map between oriented surfaces is a generalization of the winding number, defined for mappings between 1-dimensional spheres. If $S$ and $S^{\prime}$ are quadrangulated oriented surfaces and $\phi: S \rightarrow S^{\prime}$ is a cellular map, then the degree of $\phi$ can be defined as follows. Let $F$ be an arbitrary face in $S^{\prime}$ and let $n_{F}^{+}$be the number of faces in $S$ that map onto $F$ preserving the orientation, and let $n_{F}^{-}$be the number of faces mapped onto $F$ reversing their orientation. The value $n_{F}^{+}-n_{F}^{-}$is independent of the choice of $F$ and is called the degree of $\phi$; see $[20]$.
} 
odd quadrangulations also shows up in their face subdivisions $T(Q)$. It has been proved by Hutchinson et al. [7] that in an odd quadrangulation $Q$ of the projective plane, a vertex coloring with any number of colors gives rise to a four-colored face. This has been generalized to odd quadrangulations of arbitrary non-orientable surfaces in [1]; for some further extensions see also [11]. This shows that the face subdivision triangulation $T(Q)$ does not admit a local 4-coloring, and its local chromatic number is at least 5 . Since all added vertices can be colored with the same color, we also conclude that $\psi(T(Q)) \leq \psi(Q)+1$.

Theorem 7.1 If $Q$ is an odd quadrangulation of a non-orientable surface and $T(Q)$ is its face subdivision, then the local chromatic number of $T(Q)$ is at least five.

There is another family of triangulations of surfaces that exhibits unusual chromatic behavior - a simple local condition forces the chromatic number to be at least 5 despite the fact that these graphs seem to be "almost 3-colorable". Let $T$ be a triangulation of some surface such that all its vertices except two have even degree, and the two vertices of odd degree are adjacent. Fisk [5] proved that $T$ cannot be 4-colored. See [14, Section 8.4] for further details.

Here we observe that the above result of Fisk can be extended to local colorings as well.

Theorem 7.2 Let $T$ be a triangulation of some surface such that all its vertices except two have even degree. If $c$ is a local 4 -coloring of $T$, and $x$ and $y$ are the two vertices of odd degree, then $c(x)=c(y)$ and the set of three colors used on the neighbors of $x$ and of $y$, respectively, is the same. In particular, if $x$ and $y$ are adjacent, then $T$ has local chromatic number at least five.

Proof. We may assume that $c(V(T))=\{1,2, \ldots, m\}$. For every $i, j, k \in\{1,2, \ldots, m\}$, let $T_{i j k}$ be the set of facial triangles whose vertices are colored $i, j, k$, and let $V_{i}^{j k}$ be the set of vertices of color $i$ that are incident with a triangle in $T_{i j k}$.

If $v \in V(T)$, then the set of facial triangles containing $v$ determines a cycle $C$ (the link of $v$ ) passing through all the neighbors of $v$. Since $c$ is a local 4-coloring, it determines a 3-coloring of $C$, which can be viewed as a mapping of $C$ onto the cycle $C_{3}$ of length 3 . Let $w$ be the winding number of this mapping. Since $C_{3}$ has an odd number of edges, it follows that

$$
w \equiv|C|=\operatorname{deg}(v) \quad(\bmod 2) .
$$

This simple conclusion implies that the parity of the number of triangles in $T_{i j k}$ can be expressed as follows:

$$
\left|T_{i j k}\right| \equiv \sum_{u \in V_{i}^{j k}} \mathrm{deg}(u) \equiv \sum_{v \in V_{j}^{i k}} \mathrm{deg}(v) \equiv \sum_{z \in V_{k}^{i j}} \mathrm{deg}(z) \quad(\bmod 2) .
$$

There are distinct colors $i, j, k$ such that $x \in V_{i}^{j k}$ and $y \notin V_{j}^{i k}$. The second congruence in (2) can hold only when $y \in V_{i}^{j k}$ since $x$ and $y$ are the only vertices of odd degree and the sum of degrees of vertices in $V_{j}^{i k}$ is even. This shows that $x$ and $y$ have the same color and the same set of colors in their neighborhood. 


\section{Concluding remarks}

Quadrangulations can be classified into four types according to the homology of their odd cycles. Let us describe this refined classification of even/odd quadrangulations more closely. Let $G$ be a graph that is embedded in a surface $S$ such that all facial walks are of even length. Then it is easy to see that the lengths of any two closed walks in $G$ that are homologous (with respect to the $\mathbb{Z}_{2}$-homology $H_{1}\left(S, \mathbb{Z}_{2}\right)$ ) have the same parity. The parities of walks in different homology classes thus determine a homomorphism $\phi: H_{1}\left(S, \mathbb{Z}_{2}\right) \rightarrow \mathbb{Z}_{2}$, which is called the cycle parity map of $G$. If $S$ is a non-orientable surface of genus $g$, then it is homeomorphic to the connected sum of $g$ projective planes $Q_{1}, \ldots, Q_{g}$, and its homology group $H_{1}\left(S, \mathbb{Z}_{2}\right) \cong \mathbb{Z}_{2}^{g}$ is generated by 1 -sided cycles $\alpha_{i}$ in $Q_{i}, i=1, \ldots, g$. In particular, $\phi$ can be represented by the $g$-tuple $\left(\phi_{1}, \ldots, \phi_{g}\right)$, where $\phi_{i}=1$ if $Q_{i}$ contains a closed walk in $G$ of odd length, and $\phi_{i}=0$ otherwise. If $\phi_{1}=\phi_{2}=\phi_{3}=1$ and $\phi_{4}=0$, then we can replace the chosen basis $\alpha_{1}, \ldots, \alpha_{4}$ of the homology group by $\alpha_{1}^{\prime}=\alpha_{1}+\alpha_{2}+\alpha_{3}, \alpha_{2}^{\prime}=\alpha_{1}+\alpha_{2}+\alpha_{4}, \alpha_{3}^{\prime}=\alpha_{1}+\alpha_{3}+\alpha_{4}$, and $\alpha_{4}^{\prime}=\alpha_{2}+\alpha_{3}+\alpha_{4}$, respectively. It is easy to see that $\alpha_{1}^{\prime}, \ldots, \alpha_{4}^{\prime}, \alpha_{5}, \ldots, \alpha_{g}$ can be represented by disjoint 1-sided simple closed curves in $S$ that generate $H_{1}\left(S, \mathbb{Z}_{2}\right)$. Moreover, since $\phi$ is a homomorphism, it follows that the parity map representation changes from $(1,1,1,0, \ldots)$ to $(1,0,0,0, \ldots)$ under the new generating set. This shows that there is a representation of $\phi$ in one (and precisely one) of the following four forms:

$$
\begin{array}{ll}
\Phi^{0}=(0,0,0, \ldots, 0), & \Phi^{1}=(1,0,0, \ldots, 0), \\
\Phi^{2}=(1,1,0, \ldots, 0), & \Phi^{3}=(1,1,1, \ldots, 1) .
\end{array}
$$

We say that $G$ is of type $\Phi^{i}(i \in\{0,1,2,3\})$ if its cycle parity map can be represented by $\Phi^{i}$. We refer to [16] or [15] for a similar treatment with more details.

It is clear from the definition that $Q$ is of type $\Phi^{3}$ if and only if every 1-sided closed walk has odd length. (It is a corollary of this that every 2 -sided closed walk has even length.) Let us observe that the quadrangulation $G_{1}^{\prime}$ of the genus 5 non-orientable surface constructed in Section 5 is of type $\Phi^{3}$. This can be proved as follows. First we observe that $G_{1}^{\prime}$ can be represented by means of local rotations and the signature (cf. [14]). The edges of negative signature are the following ones:

$$
\begin{array}{llll}
(2,35)(3,26) & (2,36)(3,25) & (5,26)(6,35) & (5,36)(6,25) \\
(3,14)(4,35) & (3,14)(4,36) & (1,26)(6,14) & (1,36)(6,14) \\
(1,25)(5,14) & (1,35)(5,14) & (2,14)(4,25) & (2,14)(4,26)
\end{array}
$$

If we remove all these edges, we get a bipartite spanning subgraph $G_{1}^{\prime \prime}$ of $G_{1}^{\prime}$, and each removed edge joins two vertices that belong to the same bipartite class in $G_{1}^{\prime \prime}$. This shows that all 1-sided closed walks (i.e. those that traverse an odd number of edges with negative signature) in $G_{1}^{\prime}$ have odd length. Thus, $G_{1}^{\prime}$ is of type $\Phi^{3}$.

We have shown that odd quadrangulations of non-orientable surfaces of genus at most four have local chromatic number at least four and that for every surface of genus at least five, there are examples for which this no longer holds. Let us observe that a quadrangulation is odd if and only if it is either of type $\Phi^{1}$ (for arbitrary genus) or it is of type $\Phi^{3}$ when the genus is odd. As shown above, our example $G_{1}^{\prime}$ of an odd quadrangulation of genus 5 that admits a local 3 -coloring is of type $\Phi^{3}$. When producing quadrangulations of higher genera, we can switch 
to type $\Phi^{1}$ (by repeatedly inserting three diagonals into hexagonal faces as done in the proof of Theorem 1.4(ii)). But we can also stay within the type $\Phi^{3}$ by taking a quadrangulation of type $\Phi^{3}$ of odd genus $g$ that admits a local 3-coloring, such that on two adjacent faces only two colors are used, and then replace those two faces by making a connected sum with a 3 -colorable quadrangulation (minus an edge $e$ ) of the Klein bottle of type $\Phi^{2}$ in which the two faces sharing the edge $e$ are 2-colored. This gives a quadrangulation of genus $g+2$ of type $\Phi^{3}$ that admits a local 3-coloring, and leaves only one unresolved case - type $\Phi^{1}$ on the non-orientable surface of genus 5 .

Question 8.1 Is there a quadrangulation of type $\Phi^{1}$ of the non-orientable surface of genus 5 that admits a local 3-coloring?

Acknowledgements. We thank Gábor Elek for his help in finding the papers of Baudisch in the literature and for related conversations. Long discussions with László Fehér were essential for clarifying the reasoning in Section 6. Help from Gábor Moussong is also gratefully acknowledged.

\section{References}

[1] D. Archdeacon, J. Hutchinson, A. Nakamoto, S. Negami, K. Ota, Chromatic numbers of quadrangulations on closed surfaces, J. Graph Theory, 37 (2001), no. 2, 100-114.

[2] A. Baudisch, Kommutationsgleichungen in semifreien Gruppen, Acta Math. Acad. Sci. Hungar., 29 (1977), no. 3-4, 235-249.

[3] A. Baudisch, Subgroups of semifree groups. Acta Math. Acad. Sci. Hungar., 38, (1981), no. $1-4,19-28$.

[4] P. Erdős, Z. Füredi, A. Hajnal, P. Komjáth, V. Rödl, Á. Seress, Coloring graphs with locally few colors, Discrete Math., 59 (1986), 21-34.

[5] S. Fisk, The non-existence of colorings, J. Combin. Theory Ser. B, 24 (1978), 247-248.

[6] A. Gyárfás, T. Jensen, M. Stiebitz, On graphs with strongly independent color-classes, J. Graph Theory, 46 (2004), 1-14.

[7] J. Hutchinson, R. B. Richter, P. Seymour, Colouring Eulerian triangulations, J. Combin. Theory Ser. B, 84 (2002), 225-239.

[8] W. S. Massey, Algebraic Topology: An Introduction, Harcourt, Brace and World, 1967.

[9] J. Matoušek, Using the Borsuk-Ulam theorem. Lectures on topological methods in combinatorics and geometry. Written in cooperation with A. Björner and G. M. Ziegler, Universitext, Springer-Verlag, Berlin, corrected and updated 2nd printing, 2007.

[10] J. Matoušek, G. M. Ziegler, Topological lower bounds for the chromatic number: A hierarchy, Jahresber. Deutsch. Math.-Verein., 106 (2004), no. 2, 71-90, arXiv:math.CO/0208072. 
[11] B. Mohar, Quadrangulations and 5-critical graphs on the projective plane, in "Topics in Discrete Mathematics," M. Klazar, J. Kratochvíl, M. Loebl, J.Matoušek, R. Thomas, P. Valtr (Editors), Springer, 2006, pp. 565-580.

[12] H. Kneser, Die kleinste Bedeckungszahl innerhalb einer Klasse von Flächenabbildungen, Math. Ann., 103 (1930), 347-358.

[13] B. Mohar, P. D. Seymour, Coloring locally bipartite graphs on surfaces, J. Combin. Theory Ser. B, 84 (2002), no. 2, 301-310.

[14] B. Mohar, C. Thomassen, Graphs on surfaces, Johns Hopkins University Press, Baltimore, MD, 2001.

[15] A. Nakamoto, S. Negami, K. Ota, Chromatic numbers and cycle parities of quadrangulations on nonorientable closed surfaces, Discrete Math., 285 (2004), 211-218.

[16] S. Negami, A. Nakamoto, Diagonal transformations of graphs on closed surfaces, Sci. Rep. Yokohama Nat. Univ. Sec. I, 40 (1993) 71-97.

[17] G. Simonyi, G. Tardos, Local chromatic number, Ky Fan's theorem, and circular colorings, Combinatorica, 26 (2006), 587-626, arxiv:math.CO/0407075.

[18] G. Simonyi, G. Tardos, S. T. Vrećica, Local chromatic number and distinguishing the strength of topological obstructions, Trans. Amer. Math. Soc., 361 (2009), no. 2, 889-908, arxiv:math.CO/0502452.

[19] D. A. Youngs, 4-chromatic projective graphs, J. Graph Theory, 21 (1996), 219-227.

[20] H. Zieschang, E. Vogt, H.-D. Coldewey, Surfaces and planar discontinuous groups, Lecture Notes in Mathematics, 835, Springer-Verlag, Berlin, Heidelberg, New York, 1980. 